\title{
Quantitative analysis of Guided Wave in Dielectric Logging through Numerical Simulation
}

\author{
Bin Wang, Zixuan Huang, Lanchang Xing, Member, IEEE, Liyun Lao, Zhoutuo Wei and Xinmin Ge
}

\begin{abstract}
A good knowledge of the electromagnetic (EM) wave propagation behaviors in dielectric logging (DL) and borehole radar (BHR) surveying is critically important for optimization of tool design and implementation, and interpretation of the acquired logging data, as well as understanding the influences of the dielectric permittivity and conductivity of the formation on the EM waves. This paper reported a novel method for the numerical simulation and analysis of the guided wave (GW) propagating along a metallic pipe in a typical DL configuration. A numerical simulation with the 3-D finite-difference time-domain (FDTD) method was applied to the broadband DL tool to obtain the wavefield and responses of the receiver. By monitoring the wave attenuation along the metallic drill collar, the intensity of the GW and loss factor can be determined. The coupling efficiency of the GW can be obtained when the total power emitted from the transmitting antenna is known. Simulation results revealed that the coupling efficiency of the GW changes with the water saturation of the formation and frequency. The simulation also suggest, by installing a slope structure adjacent to the transmitting antenna, the energy coupled into the GW could be reduced at different levels. Finally, the relationship between the received signals' amplitude and GW's coupling efficiency showed the quantified contribution of the $G W$ to the received signal.
\end{abstract}

Index Terms - dielectric logging, guided wave, single line waveguide, finite-difference time-domain (FDTD)

\section{INTRODUCTION}

$\mathrm{D}$ IELECTRIC logging was introduced in the 1970's to evaluate the fluid saturation around a borehole, one of the key parameters for subsurface sensing. With engineering advancements, new DL tools have been developed to operate either in some discrete frequencies, or with EM pulses over a wide band from dozens of $\mathrm{MHz}$ to $\mathrm{GHz}$. They are capable of providing a permittivity dispersion measurement over a wider detection range, due to the distinct propagating modes of EM waves at different frequencies.[1-3].

A generic DL device consists of transmitters and receivers, and the waves picked up by the receivers can be decomposed into two parts: the lateral wave $(\mathrm{LW})$ and guided wave $(\mathrm{GW})[4$,

This research was jointly supported by National Natural Science Foundation of China (41704124), the Fundamental Research Funds for the Central Universities (20CX05005A and 18CX02112A), Shandong Provincial Natural Science Foundation (ZR2019MEE095 and ZR2017BEE026), and the Major Scientific and Technological Projects of CNPC (ZD2019-184-001). Thanks to Dr. Edward C. Mignot, Shandong University, for linguistic advice. (Corresponding author: Bin Wang)

Bin Wang, Zixuan Huang, Lanchang Xing is with the College of Control Science and Engineering, China University of Petroleum (East China), 266580, China (e-mail: wangbin2015@upc.edu.cn; 2232626898@qq.com; xinglc@upc.edu.cn).

Liyun Lao is with the School of Water, Energy, and Environment, Cranfield University, MK43 0AL, U.K. (e-mail: 1.lao@cranfield.ac.uk).

Zhoutuo Wei, Xinmin Ge is with the School of Geosciences, China University of Petroleum (East China), 266580, China (e-mail: weizt@upc.edu.cn; gexinmin2002@upc.edu.cn).

5]. While the GW propagates along the metallic pipe, the LW radiates into the formation surrounding the borehole, being modulated in amplitude and phase that are directly related to the complex permittivity of the formation. Due to a large contrast among the permittivities of water, oil and rock, the tool makes a direct measurement of the fluid saturation in the formation, independent of salinity. By modelling the drill pipe and surrounding media as a cylindrically concentric system, and simplifying the antennas as slotted apertures, the EM field from the GW at the receivers and the wave travel time can be analytically determined. Similarly the above can be applied to borehole radar (BHR) survey[6-8], and the simulation results suggested that the GW can still exist without borehole annulus. However, in reality a practical logging tool is too complicated to model analytically. Currently in DL and BHR practices the GW behaviors can only be qualitatively evaluated or analyzed with the use of over-simplified models, which could result in significant uncertainties.

In this paper, a method for quantitative interpretation of the GW in DL based on numerical simulation and the theory of surface wave theory was reported. Total energy flux emitted by the transmitter is estimated by using the FDTD. The local energy flux in the GW at some distance from the transmitter is estimated and its loss factor can then be determined. Further by normalizing the total initially emitted energy, the coupling efficiencies of the $\mathrm{GW}$ with different formation configurations and logging tool structures can be obtained. The simulation results are used to inform the design of DL and BHR kit.

\section{Configuration Of The Analysis Method}

The metallic pipe connected to the transmitter in the formation can act as a single line waveguide supporting an infinity of radiation modes and guided mode, which has a relatively low loss hence can sustain the propagation $[9,10]$.

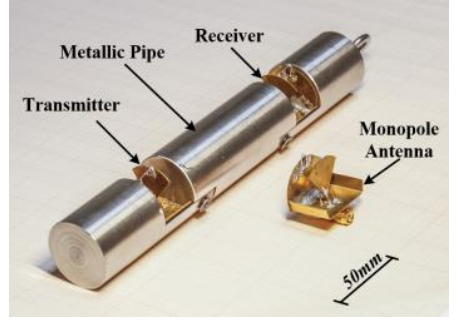

Fig.1. Photograph of the practical broadband DL prototype. The monopole antenna serving as the transmitter and receiver is shown at the right.

In this study, sector monopole ultra-wide band (UWB) antennas with a resistance and capacitance series loading served as the transmitter and receiver. It was fed through a SMA connector from the bottom, and the structural details of the tool were presented in $[3,11]$. A photograph of the DL tool with the monopole UWB antennas is presented in Fig.1. This type of DL tool was firstly proposed in our previous work, and also the GW was presented in the EM field pattern with the tool operating[3]. 
An insulated collar was implied to help to investigate the influence factors to the coupling of $\mathrm{GW}$, and this design was qualitatively proved to promote the launching of LW[11]. In this study, the mechanism of GW will be further investigated, while the power and coupling efficiency of GW can be calculated precisely with the numerical method.

As shown in Fig.2, to establish the calculation and analysis method of the GW, numerical models were first set up based on the practical structure, and the EM wave propagating process was completely simulated by the FDTD method. The total EM energy emitted by the transmitter was obtained by integration of the energy flux at the monitoring cylinder encompassing the transmitter, which is also shown in Fig.2. All the EM waves launched into the formation were divided into two parts: the GW propagating along the pipe and the LW comprising all the other power except in the GW. A sequence of monitoring planes perpendicular to the drilling pipe were virtually generated equidistantly along the pipe and numbered $1 \sim \mathrm{n}$ away from the transmitter. The normal components of the energy flux vector at those planes were integrated among the whole simulation procedure, and then the energy flowing past the specific plane in the frequency domain was acquired with Fourier transform. By differencing the energy at each two adjacent planes, and dividing it by the distance of the planes, the attenuation coefficient between the two planes was calculated in $\mathrm{dB} / \mathrm{m}$, for example:

$$
A_{s}(f)_{h-1}=10 \log \left(\frac{W_{h-1}(f)}{W_{h}(f)}\right) / d
$$

$A_{s}(f)_{h-l}$ is the simulated attenuation coefficient at frequency $f$ between $h_{t h}$ and $(h-1)_{t h}$ monitoring planes, $W_{h}(f), W_{h-l}(f)$ and $d$ are the integrated energy flux at this two monitoring planes and distance between them. Too large a distance between two adjacent planes always lead to a missing of details in the acquired energy flux; on the contrary, the calculation amount is increased with the amount of planes. This distance between two adjacent planes is optimized by balancing simulation efficiency and accuracy.

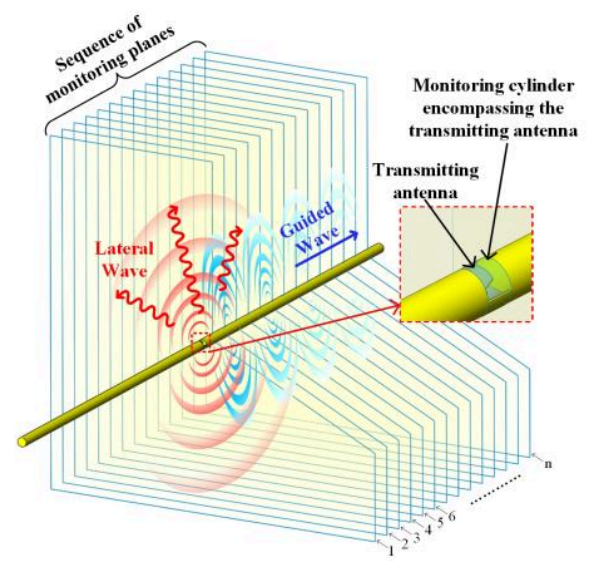

Fig.2. Schematic diagram of the numerical calculation and analysis method of the GW in the DL application. All EM energy emitted by the transmitter propagates in two ways: LW (red part) and GW (blue part), and the energy flux is calculated at the monitoring plane sequence numbered $1 \sim \mathrm{n}$. The cylindrical energy flux monitor encompasses the transmitter, as shown in the enlarged subfigure.

Perfect matching layers (PML) were set up surrounding the calculated area, so that there would be few reflecting waves at the boundaries. The concentration ratio in the radial direction of GW's energy depends on the complex permittivity of the surrounding media. Although this mode's energy theoretically extends to infinity, the field intensity dramatically decreases with the radial distance. The size of the calculated area should be appropriately large to guarantee most of GW is contained. The simulation area size was set as $2000 \mathrm{~mm} \times 2000 \mathrm{~mm} \times$ $3000 \mathrm{~mm}$ in this study, which allowed that at least $90 \%$ energy of the guide mode was included.

The propagation constant and loss factor of the GW can be calculated theoretically[10, 12]. LW quickly decreases from the transmitter, and then $A_{s}(f)$ gradually approaches the theoretical attenuation of the GW $\xi(f)$ with an increase of the distance from the transmitter. An error coefficient $\delta$ is defined, and if it meets the relationship $\delta \leq\left|\xi(f)-A_{s}(f)_{n-1}\right|$, and when the pulse has completely gone through the $n_{t h}$ monitoring plane, the simulation is terminated. In order to make the result credible, $\delta$ is generally set to be smaller than $3 \mathrm{~dB}$. In this instance, it can be considered that almost all of the EM energy passing through the $n_{t h}$ monitoring plane is in the GW. Then, for a specific frequency $f$, the energy coupled into the GW $W_{\text {guided }}(f)$ can be inversed by the following equation:

$$
W_{\text {guided }}(f)=-10^{\xi(f) \cdot d_{n}} \cdot W_{n}(f)
$$

Where $W_{n}(f)$ is the integration of energy flux at the $n_{t h}$ monitoring plane and $d_{n}$ is the vertical distance from the transmitting antenna to this plane. $W_{\text {total }}(f)$ is the total EM energy emitted by the transmitter at frequency $f$ and can be obtained with the same operation at the monitoring cylinder around the transmitter. Ultimately, the coupling efficiency of the GW $C_{\text {guided }}(f)$ can be calculated by:

$$
C_{\text {guided }}(f)=W_{\text {guided }}(f) / W_{\text {total }}(f) \times 100 \%
$$

As shown in Fig.1, the monopole antenna was fixed in a confocal cylindrical drilling pipe. To investigate the influencing factors to $C_{\text {guided }}$, a double-cambered surface was added close to the antenna in the radiating direction of the transmitter. As shown in Fig.3, the slope are tangent to the outside surface of the pipe and the transmitter's bottom surface at its two ends, and two camber are tangent to each other in the middle point of the slope. The radius of the camber $R$, the distance from baseplate to RC-Loading $h$ and the slope's length $D$ have the relationship:

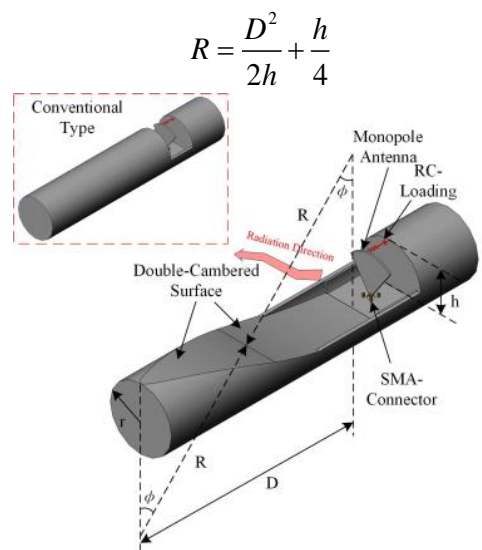

Fig.3. Metallic drilling pipe with double-cambered surface close to the 
transmitting antenna. The conventional type of transmitter and pipe without the slope surface is shown in the subfigure at top left.

The permittivity assigned to the formation $\varepsilon_{\text {formation }}^{*}$ was obtained with the complex refractive index method (CRIM)[13]:

$$
\sqrt{\varepsilon_{\text {formation }}^{*}}=(1-\phi) \sqrt{\varepsilon_{\text {sand }}}+S_{w} \phi \sqrt{\varepsilon_{\text {water }}^{*}}
$$

Quartz sand was used as the formation matrix with porosity $\Phi=40 \%$ and several water saturations $S_{w}=30 \%, 60 \%, 90 \%$ were selected as examples, where the dielectric constant of quartz sand $\varepsilon_{\text {sand }}=4$ and the dispersive complex permittivity of water $\varepsilon^{*}$ water were calculated with the Cole-Cole model[14]. The parameters of the model are summarized in Table I:

TABLE I

PARAMETERS OF THE MODEL

\begin{tabular}{cccc}
\hline \hline Parameters & Outer Diameter & Type & Electrical Properties \\
\hline Drilling Pipe & $40 \mathrm{~mm}$ & Conductor & $\sigma=7.7 \times 10^{6} \mathrm{~S} / \mathrm{m}$ \\
\hline $\begin{array}{c}\text { Annular } \\
\text { Borehole [3] }\end{array}$ & $45 \mathrm{~mm}$ & Oil mud & $\varepsilon=2+0 \mathrm{i}$ \\
\hline Formation & $\begin{array}{c}\text { Infinite with } \\
\text { PML }\end{array}$ & $\begin{array}{c}\text { Loss } \\
\text { Dielectric }\end{array}$ & $\varepsilon_{\text {formation }}^{*}$ obtained with CRIM \\
\hline \hline
\end{tabular}

\section{RESUlts AND ANALYSIS}

First, a numerical model of a conventional tool and formation with $S_{w}=30 \%$ was built. As shown in Fig.4. A modulated Gaussian pulse with a central frequency at $1.5 \mathrm{GHz}$ and operation band $0.5-2.5 \mathrm{GHz}$ was preset as the excitation signal.
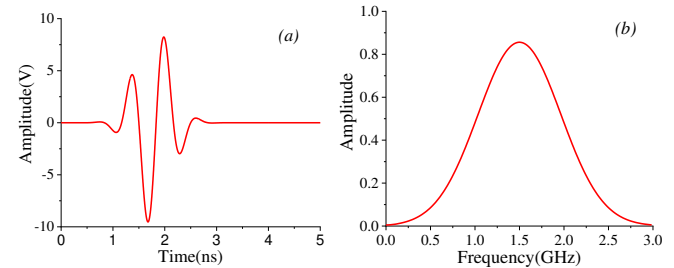

Fig.4. The excitation signal of the transmitting antenna, (a) waveform in the time domain (b) amplitude spectrum in the frequency domain.

As shown in Fig.5(a)-(c), the propagation process of the EM wave can be observed by time domain simulation. Amplitudes of the LW and GW began to differ in the near field area of the antenna, 3ns after the pulse was emitted by the transmitter. As the transmission distance increased, the $\mathrm{GW}$ gradually dominated, although it was weakened at the same time. The wave speed of the two were almost the same, and the wavefront of the LW was analogous to a spherical wave. By applying a time integral and Fourier transform, the distribution of field components could be drawn in the frequency domain. As shown in Fig.5(d), with $1.5 \mathrm{GHz}$ as an example, strong directivity was found in the LW, for the antenna was unidirectional. Also, the LW concentrated around the transmitter. The GW was axially symmetric, and the majority of its energy converged in the area close to the metallic pipe. It exponentially decayed in the radial direction, which matched the surface wave theory. An energy flux monitor sequence of 31 planes with $50 \mathrm{~mm}$ intervals was installed as shown in Fig.5(d). It was observed that there was almost no $\mathrm{LW}$ at ten planes from the right side.

For a model with $S_{w}=30 \%, A_{s}$ were calculated with (1), and are presented in Fig.6, the $A_{s}(f)$ in frequencies from $0.5 \mathrm{GHz}$ to $2.25 \mathrm{GHz}$ are a function of the distance to the transmitter. $\xi$ became bigger as frequency $f$ increased, and as the monitoring plane moved away from the transmitter, and the calculated $A_{s}$
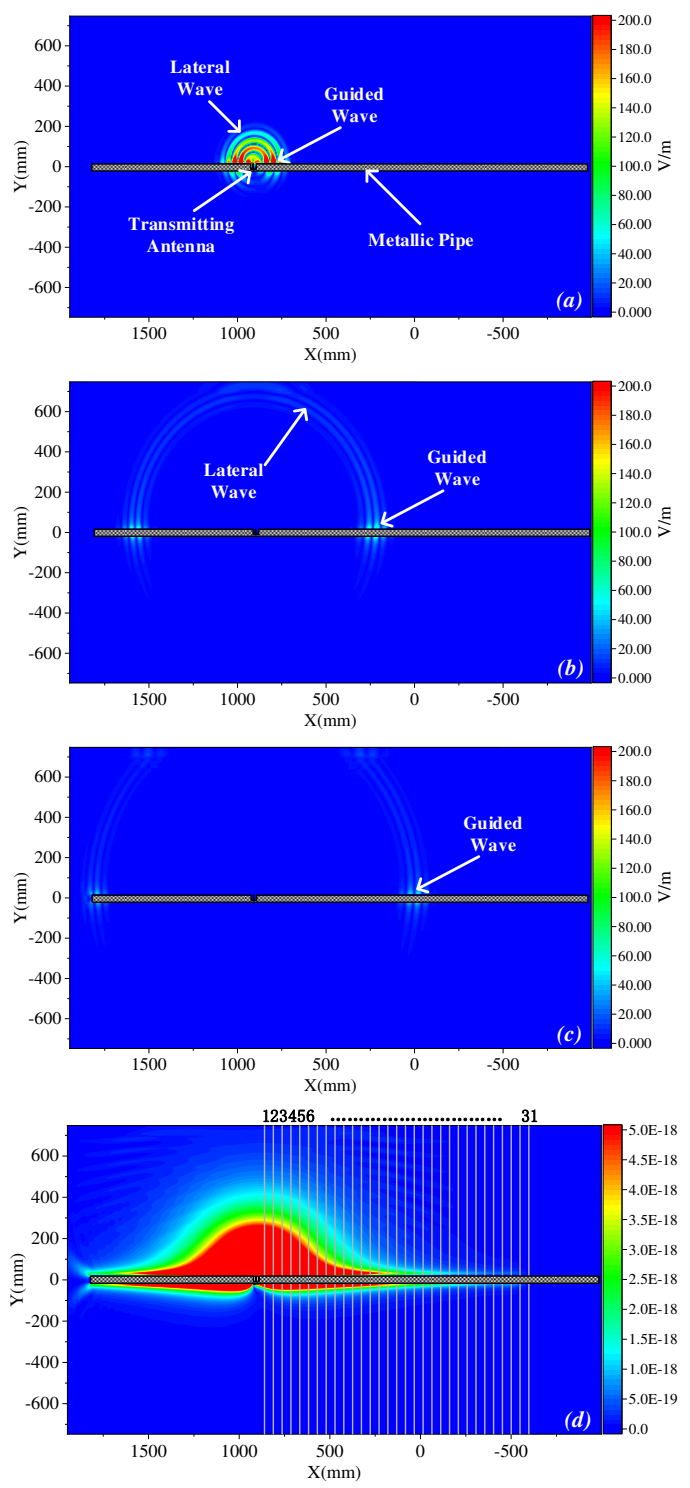

Fig.5. Photos of the E-field at different times (a)3ns, (b)8ns, (c)10ns after the stimulation of the signal; (d) the E-field at $1.5 \mathrm{GHz}$, and the side projection of monitoring planes from 1-31 are illustrated as grey lines.

gradually approached the $\xi$. The $\delta$ was set as $1 \mathrm{~dB}$, and the distance varied with frequency at which $\delta \leq\left|\xi(f)-A_{s}(f)_{n-1}\right|$ was met, because the elements of the $\mathrm{LW}$ and $C_{\text {guided }}$ are diverse at difference frequencies.

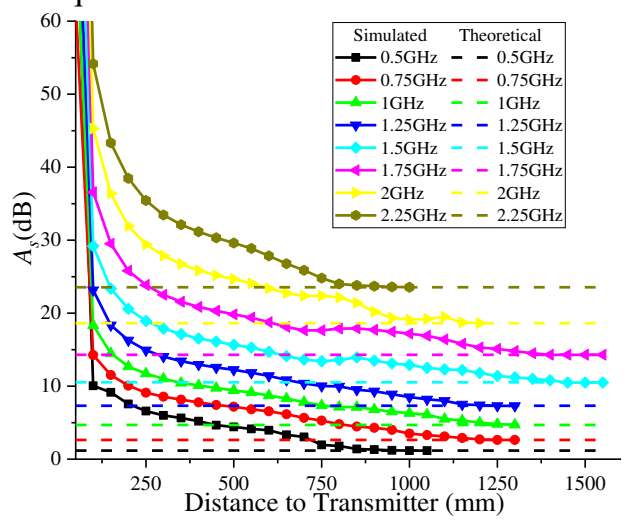

Fig.6. $A_{s}(f)$ are shown as solid line with symbols, and $\xi(f)$ are shown as dashed line at eight frequencies in a range from $0.5 \mathrm{GHz}$ to $2.25 \mathrm{GHz}$. The $A_{s}(f)$ gradually approached $\xi(f)$ as the distance to the transmitter increased, and at 
different distances, the differences between them are smaller than $\delta=1 \mathrm{~dB}$.

When the LW was depleted at the specific monitoring plane, $A_{s}$ approximately equaled $\xi$, and the $W_{\text {guided }}$ and $C_{\text {guided }}$ were calculated with (2) and (3). As shown in Fig.7, the $C_{\text {guided }}(f)$ not only varied with frequency, but also depended on $S_{w}$. The maximum value of the $C_{\text {guided }}$ drifted to a lower frequency, and at the same time $C_{\text {guided }}$ integrally declined over $0.75 \mathrm{GHz}$, as the $S_{w}$ increased. To investigate the influence of the shape of the pipe, another model of tool with a slope $(D=50 \mathrm{~mm})$ was used as the control group. The corresponding $C_{\text {guided }}$ were smaller than those obtained with the conventional pipe, however the dependence on $f$ and $S_{w}$ of $C_{\text {guided }}$ barely changed. The dielectric properties of the outer media can change the fluctuation of the $C_{\text {guided }}$ in the frequency domain, while the magnitude of $C_{\text {guided }}$ changes with the structure of the pipe.

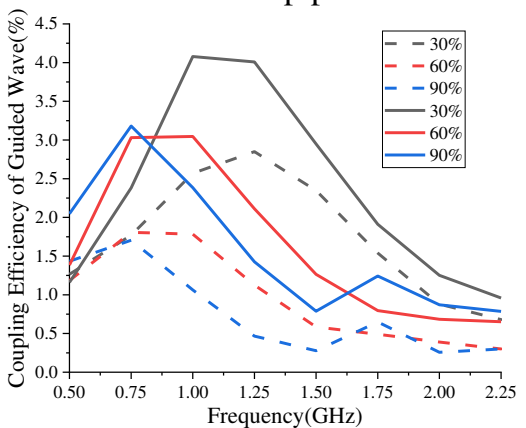

Fig.7. $C_{\text {guided }}(f)$ varied with frequency and water saturation $S_{w}$. The solid and dashed lines represent results of the conventional tool and tool with slope.

Furthermore, to validate the effect of the pipe type, the model containing pipes with slopes of different $D$ were simulated, setting $S_{w}=60 \%$ and maintaining the other parameters as the same. As shown in Fig.8, $C_{\text {guided }}$ corresponding to the pipes with a slope were all smaller than the conventional type pipe. The $C_{\text {guided }}$ inversely varied with the $D$, and the relationship was not linear. The decline rate of the $C_{\text {guided }}$ gradually slowed down as $D$ increased, and thus they were significantly close when the $D$ were $150 \mathrm{~mm}$ and $200 \mathrm{~mm}$. The majority of the discrepancy was observed in the frequency band from $0.75 \mathrm{GHz}$ to $1.5 \mathrm{GHz}$. This is because the wavelength in this frequency band is very close to the size of the transmitter and the slope structure, and thus the mode components of the launched EM wave was sensitive to the structure of the tool. The slopes spoiled the cylindrical waveguide, especially when it was close to the transmitter.

Referring to Fig.5, the amplitude inside the nearfield of the antenna was critically bigger than other regions, and it can be inferred that the structure inside this area may significantly affect the modes coupling. Slopes with $D$ larger than $150 \mathrm{~mm}$ had a slight difference inside the antenna nearfield, so it made the $C_{\text {guided }}$ approximately the same.

As shown in Fig.9, corresponding to different pipe structures, there was a tiny discrepancy in $W_{\text {total }}$. The maximum discrepancy was reached at $1.5 \mathrm{GHz}$. This was reasonable, because $1.5 \mathrm{GHz}$ was the center frequency of the excitation signal. In addition, the actual wavelength of $1.5 \mathrm{GHz}$ was approximately $50 \mathrm{~mm}$ which was nearly equal to the diameter of the drilling pipe and the size of the antenna cavity, when $S_{w}=60 \%$. Changes in structure very close to the monopole

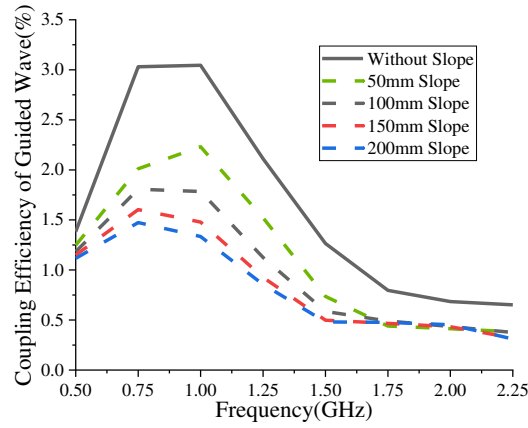

Fig.8. $C_{\text {guided }}$ varied with the length of double-cambered slope, when the water saturation $S_{w}=60 \%$ and they are drawn as dashed lines. The solid line represents the coupling efficiency of a structure without a slope.

can alter the radiation efficiency of the antenna, although this impact was very limited according to this case. Referring to the $W_{\text {total }}$, the declination of $C_{\text {guided }}(f)$ with $D$ increase fundamentally resulted from the decrease of the absolute energy coupled into the surface mode.

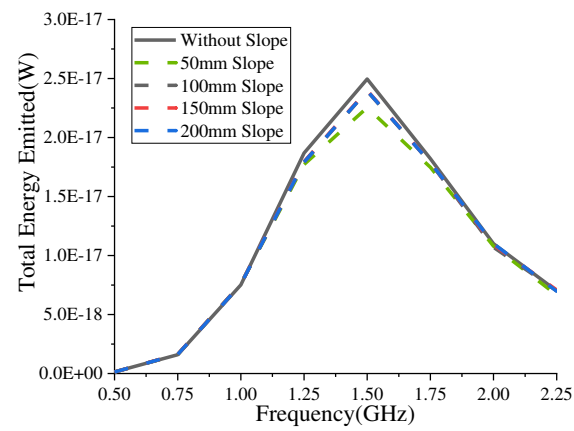

Fig.9. $W_{\text {total }}$ varied with frequency, when $S_{\mathrm{w}}=60 \%$ and they are drawn as dashed lines. The solid line represents the energy of a structure without a slope.

To investigate the contribution of the $\mathrm{GW}$ to the received signals, some models of $S_{w}=60 \%$ with two antennas towards each other's main radiating direction were established. The distance of the receiver and transmitter was $500 \mathrm{~mm}$, and the amplitude spectra of the received signals are shown in Fig.10. It was found that the magnitude decreased with the $D$, and their

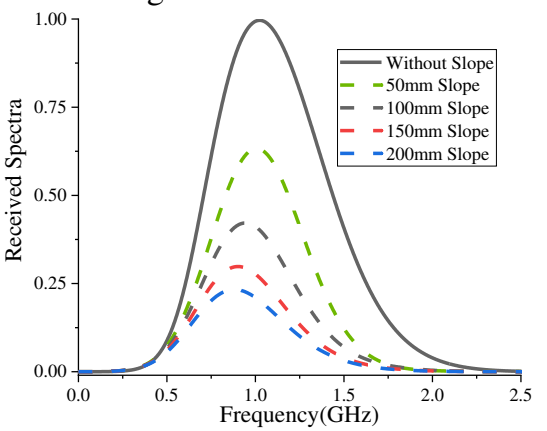

Fig.10. Spectra of the received signals, with $S_{w}=60 \%$ and the distance between receiving and transmitting antenna as $500 \mathrm{~mm}$.

center frequency drifted to a lower band. The analogous phenomenon can be found in the $C_{\text {guided }}$ in Fig.8, and therefore we hypothesize that the received signals may potentially depend on $C_{\text {guided }}$. Even though there were distinctions between the frequency of the maximum amplitude of them, it is because the received signals also depend on frequency components of the excitation signals and their propagation characteristics.

To quantify the contribution of the $\mathrm{GW}$ in the received 
signals, as shown in Fig.11, the relationship between $C_{\text {guided }}$ and the amplitude are illustrated. Regardless of frequency, the amplitude and $C_{\text {guided }}$ were positively related, however the increasing rate of amplitude dependents on the frequency.

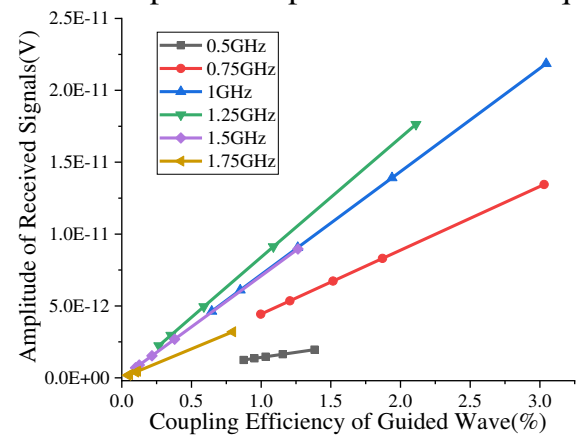

Fig.11. The relationship between $C_{\text {guided }}$ and the amplitude of the received signals with $S_{w}=60 \%$. The simulated data are symbols, and the corresponding linear fitted results are solid lines with the same color. The symbols from right to left are in order: without slope, $D$ of $50 \mathrm{~mm}, 100 \mathrm{~mm}, 150 \mathrm{~mm}$, and $200 \mathrm{~mm}$.

By applying linear fitting to the results, a group of solid lines in Fig. 11 was obtained, and their slopes are shown in Table II. It was maximized at $1.25 \mathrm{GHz}$, and the declination was almost symmetrical at higher and lower frequencies. This is because that at $1.25 \mathrm{GHz}$ the received signals depend more on the $\mathrm{GW}$ than the other frequencies. In other words, $C_{\text {guided }}(f)$ at $1.25 \mathrm{GHz}$ make more differences in spectra of received signals. For lower frequencies, e.g. $0.5 \mathrm{GHz}$, the wavelength was critically larger than the structure size, so the details of the pipe was electrically small objects. The fitted slope at $0.5 \mathrm{GHz}$ was only $17 \%$ of that at $1.25 \mathrm{GHz}$, and the solid line at $0.5 \mathrm{GHz}$ was nearly horizontal, which means $C_{\text {guided }}$ had almost no effect on the received signal. For higher frequency, e.g. $1.75 \mathrm{GHz}$, the wavelength was much smaller than the details of the tool, and thus $C_{\text {guided }}$ with slopes were negligible compared to the conventional type. Also, there was little EM energy induced by the receiving antenna at $1.75 \mathrm{GHz}$. This is because the slope structure crucially spoiled the cylindrical structures, and caused the emitted EM wave to decay to vanishing before being coupled into the surface mode. The evaluation of the GW can surely help the tool design and data analysis. For BHR, it is rational to optimize the tool design by reducing the slopes as in Fig. 11. For example spoiling the cylindrical structures can eliminate the influence on responses from GW, diminishing the artifacts in responses of BHR. For DL inversion, the proposed method can help to evaluate the weightiness of the $\mathrm{GW}$ in the receiving signals, which determine the transverse detecting depth of the tool. Moreover, the drilling pipe with slopes allows the detecting area to be focused near the borehole, improving near-borehole resolution. TABLE II

SLOPE OF THE LINEAR FITTING LINES

\begin{tabular}{ccccccc}
\hline \hline Frequency $(\mathrm{GHz})$ & 0.5 & 0.75 & 1 & 1.25 & 1.5 & 1.75 \\
\hline Slope $\times 1 \mathrm{E}-12$ & 1.42 & 4.44 & 7.18 & 8.35 & 7.09 & 4.02 \\
\hline \hline
\end{tabular}

\section{CONCLUSION}

A novel method for the quantitative evaluation of the EM wavefield in DL and BHR was presented, and the GW was adequately analyzed as well as its relationship with the responses of the receiver based on practical tool structures. Two kinds of logging tools were considered: a conventional type and the other with slope near the antenna. The propagation process of the total EM wavefield was simulated by FDTD at several typical saturations, and the energy flux was recorded at a series of monitors. The launched EM wave can be coupled with the metallic pipe to induce $\mathrm{GW}$.

The attenuations at monitoring planes gradually approached the theoretical value. Then the energy of GW could be inversed with different saturations and tool structures, and many significant conclusions were drawn. The coupling efficiency is affected by the saturation of the formation and varied with frequency, and a slope can reduce the GW to different degrees. Also, from the linear relationship between the coupling efficiency and the responses at the receivers, the contribution of the GW to the received signals can be directly evaluated. The proposed method can quantitatively analyze the GW in DL and BHR applications with complicated structure. This method is valuable for the inversion of the acquired logging data, and implementation of subsurface sensing.

\section{REFERENCES}

[1] M. Pirrone et al., "A novel approach based on dielectric dispersion measurements to evaluate the quality of complex shaly-sand reservoirs," in SPE Annual Technical Conference and Exhibition, 2011: Society of Petroleum Engineers.

[2] S. Liu and M. Sato, "Electromagnetic logging technique based on borehole radar," IEEE transactions on geoscience and remote sensing, vol. 40, no. 9, pp. 2083-2092, 2002.

[3] B. Wang, K. Li, F.-M. Kong, J. Zhao, and Y. Gao, "A Borehole Fluid Saturation Evaluation Method Using Unidirectional Monopole UWB Antenna," IEEE Geoscience and Remote Sensing Letters, vol. 12, no. 2, pp. 429-433, 2014.

[4] W. C. Chew and S. C. Gianzero, "Theoretical investigation of the electromagnetic wave propagation tool," IEEE Transactions on Geoscience and Remote Sensing, no. 1, pp. 1-7, 1981.

[5] J. Wait, "General solution for excitation by slotted aperture source in conducting cylinder with concentric layering," IEEE transactions on microwave theory and techniques, vol. 35, no. 3, pp. 321-325, 1987.

[6] L. Mao and B. Zhou, "Simulation and analysis of conductively guided borehole radar wave," IEEE Transactions on Geoscience and Remote Sensing, vol. 55, no. 5, pp. 2646-2657, 2017.

[7] B. Zhou and M. van de Werken, "Conductively guided borehole radar wave for imaging ahead of a drill bit," IEEE Geoscience and Remote Sensing Letters, vol. 12, no. 8, pp. 1715-1719, 2015.

[8] I. M. Mason, A. J. Bray, T. G. Sindle, C. M. Simmat, and J. H. Cloete, "The effect of conduction on VHF radar images shot in water-filled boreholes," IEEE Geoscience and Remote Sensing Letters, vol. 5, no. 2, pp. 304-307, 2008.

[9] G. Elmore, "Introduction to the Propagating Wave on a Single Conductor," ed, 2009.

[10] S. Ebihara, M. Sato, and H. Niitsuma, "Analysis of a guided wave along a conducting structure in a borehole " Geophysical prospecting, vol. 46, no. 5, pp. 489-505, 1998.

[11] B. Wang, K. Li, F.-M. Kong, and J. Zhao, "A dielectric logging tool with insulated collar for formation fluid detection around borehole," Journal of Applied Geophysics, vol. 119, pp. 16-24, 2015.

[12] S. Ebihara, A. Sasakura, and T. Takemoto, "HE11 Mode Effect on Direct Wave in Single-Hole Borehole Radar," IEEE Transactions on Geoscience and Remote Sensing, vol. 49, no. 2, pp. 854-867, 2010.

[13] V. L. Mironov, M. C. Dobson, V. H. Kaupp, S. A. Komarov, and V. N. Kleshchenko, "Generalized refractive mixing dielectric model for moist soils," IEEE transactions on Geoscience and Remote sensing, vol. 42, no. 4, pp. 773-785, 2004.

[14] U. Kaatze, "Complex permittivity of water as a function of frequency and temperature," Journal of Chemical and Engineering Data, vol. 34, no. 4, pp. 371-374, 1989. 